\title{
Assessment of intracranial venous blood flow after subarachnoid hemorrhage: a new approach to diagnose vasospasm with transcranial color-coded duplex sonography
}

\author{
Florian Connolly, MD, ${ }^{1}$ Stephan J. Schreiber, MD, ${ }^{1}$ Christoph Leithner, MD, ${ }^{1}$ Georg Bohner, MD, ${ }^{2}$ \\ Peter Vajkoczy, MD, ${ }^{3}$ and José M. Valdueza, MD ${ }^{4}$
}

Departments of ${ }^{1}$ Neurology, ${ }^{2}$ Neuroradiology, and ${ }^{3}$ Neurosurgery, University Hospital Charité, Berlin; and ${ }^{4}$ Neurological Center, Segeberger Kliniken, Bad Segeberg, Germany

OBJECTIVE Transcranial color-coded duplex sonography (TCCS) is a reliable tool that is used to assess vasospasm in the $\mathrm{M}_{1}$ segment of the middle cerebral artery (MCA) after subarachnoid hemorrhage (SAH). A distinct increase in blood flow velocity (BFV) is the principal criterion for vasospasm. The MCA/internal carotid artery (ICA) index (Lindegaard Index) is also widely used to distinguish between vasospasm and cerebral hyperperfusion. However, extracranial ultrasonography assessment of the neck vessels might be difficult in an intensive care unit. Therefore, the authors evaluated whether the relationship of intracranial arterial to venous BFV might indicate vasospasm with similar or even better accuracy.

METHODS Patients who presented between 2008 and 2015 with aneurysmal SAH were prospectively enrolled in the study. Digital subtraction angiography (DSA) and TCCS were performed within 24 hours of each other to assess vasospasm 8-10 days after SAH. The following different TCCS parameters were analyzed to assess vasospasm in the MCA and were compared with the gold-standard DSA parameters: 1) mean time-averaged maximum BFV $\left(V_{\text {mean }}\right)$ of the MCA, 2) peak systolic velocity (PSV) of the MCA, 3) the Lindegaard Index using $V_{\text {mean }}$ as well as PSV, and 4) a new arteriovenous index ( $\mathrm{AVI}$ ) between the MCA and the basal vein of Rosenthal using $\mathrm{V}_{\text {mean }}$ and PSV. The best cutoff values for these parameters to distinguish vasospasm from normal perfusion or hyperperfusion were calculated using receiver operating characteristic curve analysis. Sensitivity, specificity, positive predictive value, and negative predictive value as well as the overall accuracy for each cutoff value were analyzed.

RESULTS A total of 102 patients (mean age $52 \pm 12$ years) were evaluated. Bilateral MCA assessment by TCCS was successful in all patients. In 6 cases (3\%), the BFV of the basal vein of Rosenthal could not be analyzed. The AVI could not be calculated in 50 of 204 cases (25\%) because the insonation quality was very low in one of the ICAs. An AVI > 10 for $\mathrm{V}_{\text {mean }}$ and an $\mathrm{AVI}>12$ for systolic velocity provided the highest accuracies of $87 \%$ and $86 \%$, respectively. Regarding the Lindegaard Index, the accuracy was highest using a threshold of $>3$ for the mean BFV (84\%) as well as systolic BFV $(80 \%)$. BFVs in the MCA of $\geq 120 \mathrm{~cm} / \mathrm{sec}\left(\mathrm{V}_{\text {mean }}\right)$ and $\geq 200 \mathrm{~cm} / \mathrm{sec}$ (PSV) predicted vasospasm with accuracies of $84 \%$ and $83 \%$, respectively. A combined analysis of the MCA BFV and the AVI led to a slight increase in specificity $\left(\mathrm{V}_{\text {mean }}\right.$, $94 \%$; PSV, 93\%) and positive predictive value $\left(\mathrm{V}_{\text {mean }}, 88 \%\right.$; PSV 86\%) without further improvement in accuracy $\left(\mathrm{V}_{\text {mean }}\right.$, $88 \%$; PSV, 84\%).

CONCLUSIONS The intracranial AVI is a reliable parameter that can be used to assess vasospasm after SAH. Its reliability for differentiating vasospasm and hyperperfusion is slightly higher than that for the established Lindegaard Index, and this method has the additional advantage of a remarkably lower failure rate.

https://thejns.org/doi/abs/10.3171/2017.5.JNS17232

KEY WORDS subarachnoid hemorrhage; vasospasm; transcranial color-coded sonography; basal vein of Rosenthal; Lindegaard Index; vascular disorders

ABBREVIATIONS AVI = arteriovenous index; BFV = blood flow velocity; CBF = cerebral blood flow; DSA = digital subtraction angiography; ICA = internal carotid artery; $\mathrm{ICP}=$ intracranial pressure; $\mathrm{MCA}=$ middle cerebral artery; NPV = negative predictive value; $\mathrm{PPV}=$ positive predictive value; $\mathrm{PSV}=$ peak systolic velocity; ROC = receiver operating characteristic; $\mathrm{SAH}=$ subarachnoid hemorrhage; TCCS = transcranial color-coded duplex sonography; $\mathrm{TCD}=$ transcranial Doppler; $\mathrm{V}_{\text {mean }}=$ mean time-averaged maximum BFV.

SUBMITTED January 27, 2017. ACCEPTED May 30, 2017.

INCLUDE WHEN CITING Published online December 15, 2017; DOI: 10.3171/2017.5.JNS17232. 
$\mathrm{T}$ RansCRanial Doppler (TCD) ultrasonography and transcranial color-coded duplex sonography (TCCS) are both well-established methods to assess vasospasm in the acute phase after subarachnoid hemorrhage (SAH) ${ }^{4}$ Digital subtraction angiography (DSA) is the gold standard for the detection and grading of vasospasm after SAH. Sensitivity and specificity of TCD ultrasonography techniques regarding vasospasm vary widely depending on the analyzed intracranial artery and the different diagnostic criteria. ${ }^{6,9,10,17,20,24,25}$ The reliability of TCD ultrasonography in comparison with DSA is greatest in the $\mathrm{M}_{1}$ segment of the middle cerebral artery (MCA), which is also the preferred artery to study. A very high specificity of $98 \%$ for vasospasm in the MCA was found when a mean time-averaged maximum blood flow velocity (BFV) $\left(\mathrm{V}_{\text {mean }}\right)$ of $\geq 200 \mathrm{~cm} / \mathrm{sec}$ has been defined as the diagnostic criterion. However, this high cutoff value leads to poor sensitivity of $27 \%$, indicating a high number of falsenegative results. ${ }^{23}$ More commonly used is the threshold value of $\geq 120 \mathrm{~cm} / \mathrm{sec}\left(\mathrm{V}_{\text {mean }}\right)$ in the MCA, leading in the same study to a sensitivity of $88 \%$ and specificity of $72 \%$ obtained with TCD ultrasonography compared with DSA. However, the poor positive predictive value (PPV) of 55\%, indicating numerous false-positive findings, suggests that increased BFV due to cerebral hyperperfusion might be a relevant differential diagnosis. Reliable data regarding the significance and the time course of hyperperfusion after SAH are scarce. A phase of relative hypoperfusion within the first 2 weeks followed by regional or global cerebral hyperperfusion was found using serial SPECT measurements. ${ }^{2}$

An MCA/internal carotid artery (ICA) ratio of the MCA BFV to the ICA BFV (Lindegaard Index) was proposed for better discrimination between vasospasm and cerebral hyperperfusion using TCD ultrasonography. ${ }^{8}$ This index might be a useful tool, especially in patients with moderately increased BFV. During the last 2 decades, TCCS has also been used to show similar or even better diagnostic accuracy in the assessment of vasospasm. ${ }^{6,10,14,20}$ However, reliable calculation of the Lindegaard Index via duplex techniques such as TCCS might be difficult in the ICU setting. Restricted neck movements and central lines in the internal jugular vein can impede optimal placement of the linear array probe for accurate angle correction in the ICA.

Little is known about the venous hemodynamics in $\mathrm{SAH}$ patients. Intracranial venous BFV might reflect the cerebral blood flow (CBF) more reliably than the arterial BFV after SAH. ${ }^{11}$ Furthermore, patients with SAH and vasospasm who showed a decrease in BFV in the basal vein of Rosenthal had a worse clinical outcome than patients with an increase in venous BFV. ${ }^{11}$ Therefore, raised venous BFV might be an indicator of cerebral hyperperfusion.

Based on these findings, we aimed to investigate whether calculation of an intracranial arteriovenous index (AVI) between BFVs in the MCA and the basal vein of Rosenthal might improve the feasibility and accuracy of vasospasm assessment by TCCS in comparison with exclusive arterial BFV assessment or the Lindegaard Index.

\section{Methods \\ Patient Population}

Patients who presented between 2008 and 2015 with aneurysmal SAH, survived at least until Day 10, and were evaluated using both TCCS and DSA were prospectively enrolled in the study. Our institutional ethics committee provided full approval for the study. Diagnosis of SAH was based on cerebral CT scanning immediately after admission to our emergency department. DSA was performed within the first 24 hours to localize the ruptured aneurysm. Based on the localization and shape of the aneurysm, treatment was performed surgically or by endovascular intervention.

\section{Ultrasound Examinations}

All TCCS examinations were performed by the same experienced investigator (F.C.). Only patients with a sufficient transtemporal bone window for insonation of the MCA bilaterally were included. Using the transtemporal approach, serial TCCS measurements of the $M_{1}$ segment and the basal vein of Rosenthal were performed bilaterally in the midbrain plane in each patient according to standard criteria (2.5-MHz phase array probe; Power Vision 6000, Toshiba). ${ }^{22} \mathrm{~V}_{\text {mean }}$ and peak systolic velocity (PSV) were calculated in the MCA and basal vein of Rosenthal without angle correction by automatic or, in case of a weak Doppler spectrum, by manual tracing of the maximum frequency envelope of the waveform. The entire $\mathrm{M}_{1}$ segment and the distal portion of the basal vein of Rosenthal running parallel to the $\mathrm{P}_{2}$ segment of the posterior cerebral artery with a flow direction away from the probe were recorded with a 3-mm-wide sample volume (Figs. 1 and 2). The highest detectable BFVs were used for further analysis. The anglecorrected $\mathrm{V}_{\text {mean }}$ and PSV of the extracranial ICA were then assessed bilaterally using a 7.5-MHz linear-array transducer of the same duplex ultrasound machine. The ICA was insonated in a longitudinal plane distal to the carotid bulb. The Lindegaard Index was calculated bilaterally by using the $\mathrm{V}_{\text {mean }}$ and PSV separately. Then, the AVI was calculated for both sides by using the $\mathrm{V}_{\text {mean }}$ and PSV separately. All parameters (BFV of the MCA, and Lindegaard Index and AVI, both with $\mathrm{V}_{\text {mean }}$ and PSV) were compared with the gold-standard DSA parameters.

\section{Digital Subtraction Angiography}

A second selective DSA study in both ICAs was routinely performed in all patients with severe $\mathrm{SAH}$ in our hospital between Days 8 and 10 after SAH. Standard images contained an anteroposterior, a lateral, and at least one oblique view. Injection rates varied between 4 and 7 $\mathrm{ml} / \mathrm{sec}$ (manual injection) at an imaging rate of 3 frames per second (within the arterial phase). All DSA images were analyzed by an experienced neuroradiologist (G.B.). The view showing the most severe $\mathrm{M}_{1}$ segment narrowing was used for comparison with the TCCS images. All measurements were performed using the electronic calipers on the digital display station. The resolution of this method in distance measurement is $0.1 \mathrm{~mm}$. Angiographic diagnosis of vasospasm was based on widely used criteria. ${ }^{6-8,10,16,23}$ To grade the extent of vasospasm, the point 


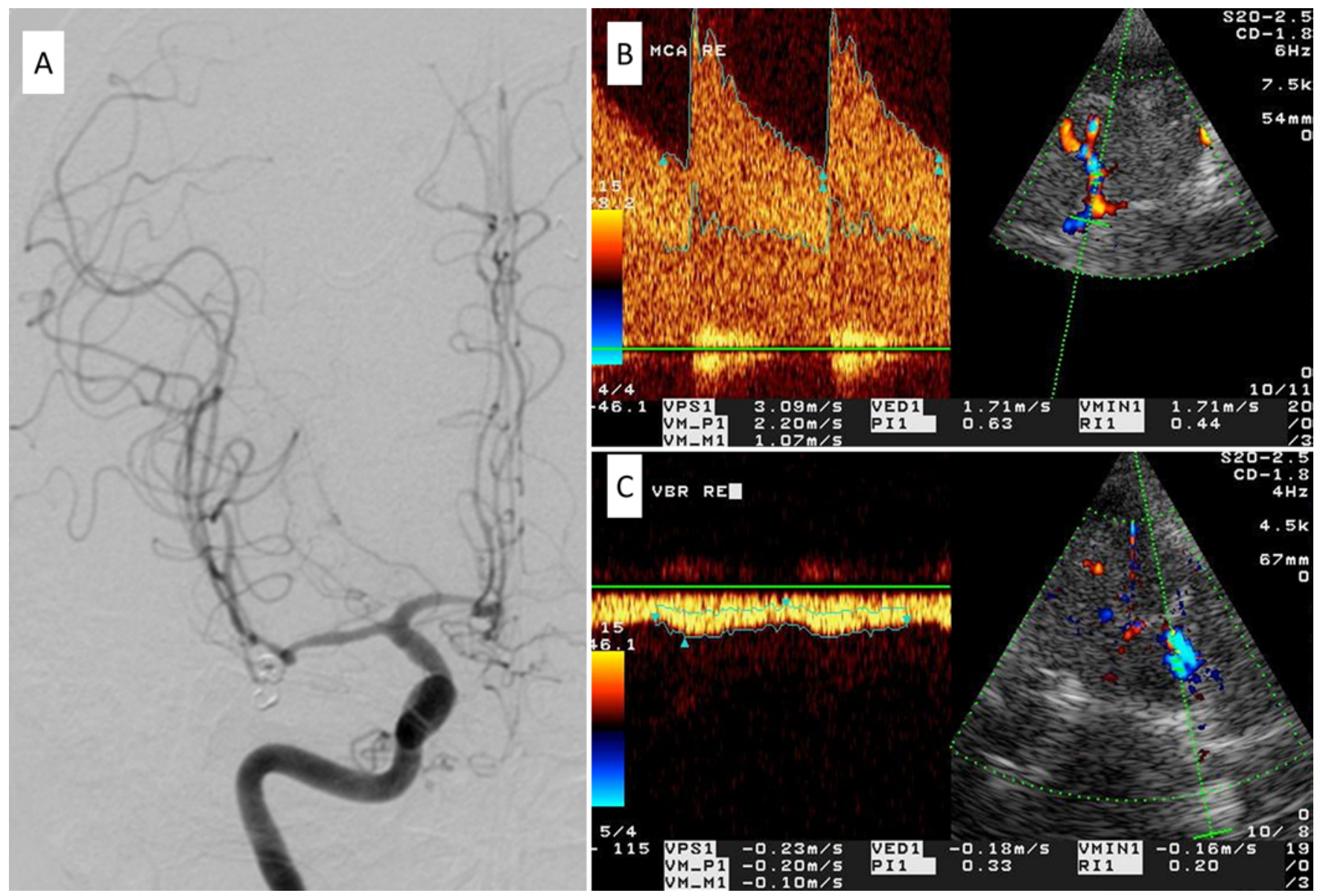

FIG. 1. Images obtained in a 52-year-old patient with moderate vasospasm in the right $M_{1}-M C A\left(M_{1}\right.$ segment of the $\left.M C A\right)$. A: Digital subtraction angiogram, right ICA injection, posteroanterior view, arterial phase. Note the vessel narrowing of the right $M_{1}$ segment. B: TCCS study (transtemporal approach), right-sided insonation, midbrain plane. Distinct increased BFV in the right $\mathrm{M}_{1}$ segment (BFV: $309 \mathrm{~cm} / \mathrm{sec} ; \mathrm{V}_{\text {mean }}: 220 \mathrm{~cm} / \mathrm{sec}$ ). C: TCCS study (transtemporal approach), right-sided insonation, midbrain plane. Slightly increased BFV in the right basal vein of Rosenthal (BFV: $23 \mathrm{~cm} / \mathrm{sec} ; V_{\text {mean }}: 20 \mathrm{~cm} / \mathrm{sec}$ ). The resulting AVI is 13.4 (PSV) and $11\left(\mathrm{~V}_{\text {mean }}\right)$, respectively. The moderately increased BFV of the basal vein of Rosenthal indicates a concomitant mild hyperemia also. Figure is available in color online only.

of maximum diameter reduction in the $\mathrm{M}_{1}$ segment was compared with the undisturbed same vessel segment on the initial DS angiogram on the day of admission. Vasospasm was quantified as none, mild $(\leq 25 \%)$, moderate $(>$ $25 \%$ to $50 \%$ ), or severe ( $>50 \%$ vessel caliber reduction). For further analysis and comparison with TCCS findings, patients were placed into one of 2 groups: those with vasospasm and those without vasospasm.

The second DSA study-performed for optimal vasospasm diagnosis-was used as the gold standard in this study for comparison analyses with the TCCS results. No intraarterial therapy for vasospasm was performed. TCCS was performed within 24 hours after DSA. The TCCS investigator and the neuroradiologist were blinded to results of the other method.

\section{Statistical Analysis}

All data are presented as the mean \pm SD. The best cutoff values for all TCCS parameters (BFV of the MCA, and Lindegaard Index and AVI, both with $\mathrm{V}_{\text {mean }}$ and PSV) for the detection of vasospasm were calculated using re- ceiver operating characteristic (ROC) curve analysis. The ROC curve was constructed by graphing the sensitivity on the ordinate as a function of the false-positive rate (1 specificity) (Fig. 3). For all cutoff values, the PPV, negative predictive value (NPV), sensitivity, specificity, and overall accuracy were assessed. A p value $<0.05$ was considered statistically significant. All statistics were calculated using IBM SPSS software (version 23, IBM Corp.).

\section{Results}

A total of 102 consecutive patients with aneurysmal SAH (age range 17-81 years, mean $52 \pm 12$ years; 68 female patients) were enrolled in the study. DSA performed on Days 8-10 revealed vasospasm of the $M_{1}$ segment in 48 patients (47\%). Vasospasm was detected in $79(39 \%)$ of $204 \mathrm{M}_{1}$ segments (40 in the right and 39 in the left segments).

$\mathrm{BFV}$ in the MCA was measured in all patients on both sides (Figs. 1 and 2). Assessment of the AVI was not possible in 11 cases $(5 \%$; on the right in 5 cases and on the left 


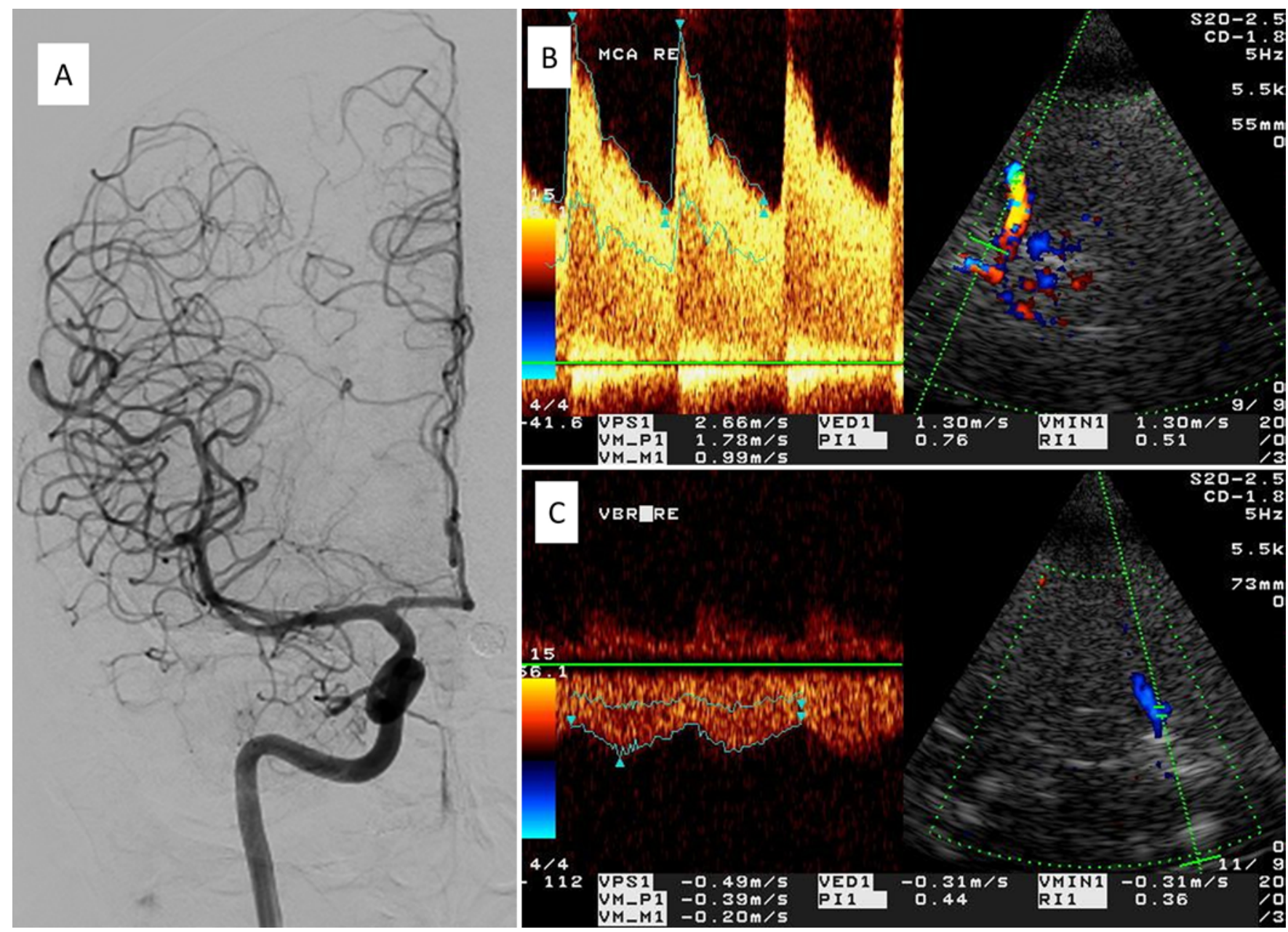

FIG. 2. Images obtained in a 49-year-old patient with cerebral hyperperfusion without vasospasm in the right $M_{1}-M C A$. A: Digital subtraction angiography, right ICA injection, posteroanterior view, arterial phase. Normal diameter of the right $\mathrm{M}_{1}$ segment. B: TCCS (transtemporal approach), right-sided insonation, midbrain plane. Increased BFV in the right $M_{1}$ segment (BFV: 266 $\mathrm{cm} / \mathrm{sec}$; $V_{\text {mean }}: 178 \mathrm{~cm} / \mathrm{sec}$ ). C: TCCS study (transtemporal approach), right-sided insonation, midbrain plane. Increased BFV in the right basal vein of Rosenthal (BFV: $\left.49 \mathrm{~cm} / \mathrm{sec} ; \mathrm{V}_{\text {mean }}: 39 \mathrm{~cm} / \mathrm{sec}\right)$. The resulting $\mathrm{AVI}$ is 5.4 (PSV) and $4.6\left(\mathrm{~V}_{\text {mean }}\right.$ ), respectively. Figure is available in color online only.

in 6 cases), whereas calculation of the Lindegaard Index failed in 42 cases $(21 \%$; on the right in 27 cases and on the left in 15 cases). ROC analysis yielded a $\mathrm{V}_{\text {mean }}>120 \mathrm{~cm} /$ $\mathrm{sec}$ and $\mathrm{PSV}>200 \mathrm{~cm} / \mathrm{sec}$ as the best cutoff values to predict vasospasm by means of BFV in the MCA (Fig. 3). Corresponding findings for the other parameters regarding the ideal cutoff were a Lindegaard Index $>3$ and an AVI $>10$ or $>12$ using $V_{\text {mean }}$ or PSV, respectively. BFV in the basal vein of Rosenthal did not differ between patients with mild and severe vasospasm $\left(\mathrm{V}_{\text {mean }}, 12 \pm 4\right.$ vs $13 \pm 4$ $\mathrm{cm} / \mathrm{sec}$; PSV, $16 \pm 4$ vs $16 \pm 6 \mathrm{~cm} / \mathrm{sec})$.

The sensitivity, specificity, PPV, NPV, and accuracy of TCCS to predict vasospasm according to the different analyzed parameters are summarized in Table 1. The greatest accuracy was found for the combined assessment of BFV in the MCA and the AVI using $\mathrm{V}_{\text {mean }}(88 \%)$, followed by the exclusive calculation of the AVI based on $\mathrm{V}_{\text {mean }}(87 \%)$. No significant differences, however, were found regarding the diagnostic accuracy of the BFV in the MCA and the Lindegaard Index (both $84 \%$ based on $\mathrm{V}_{\text {mean }}$ ).

\section{Discussion}

We assessed the ratio between intracranial arterial and venous BFVs assessed by TCCS as a new method to evaluate vasospasm after $\mathrm{SAH}$ in comparison with conventional Doppler criteria. The MCA and the basal vein of Rosenthal are probably the most appropriate vessels for calculating the AVI. Both vessels follow a stable anatomical course, hypoplasia or anatomical variants are very rare, the insonation rate is high, well-established reference values of BFV exist, and an angle correction is not necessary for reliable assessment of the BFV..$^{19,21}$ In addition, vasospasm after SAH most often affects the MCA, the artery with the most reliable data regarding the Doppler-based analysis of vasospasm. ${ }^{23}$ Furthermore, the comparison of TCCS findings and DSA as the gold standard provides the high reliability of our data.

A significant increase in BFV in the MCA and a high Lindegaard Index are the main Doppler criteria for vasospasm after SAH. However, dynamic changes in BFV are 

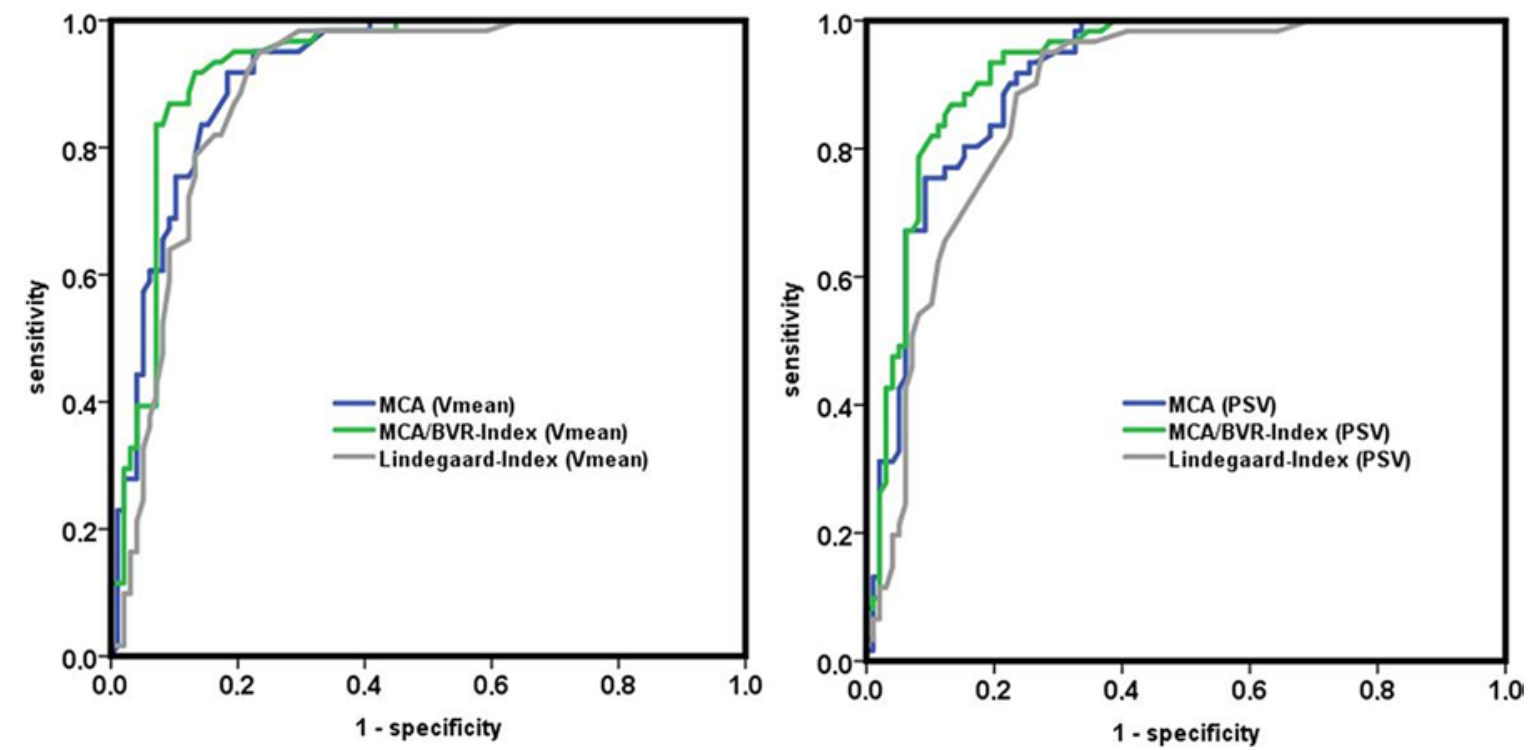

FIG. 3. Graphs showing ROC curves for diagnosis of MCA vasospasm with TCCS. Curves constructed for mean (left) and peak systolic (right) BFV in the MCA (blue lines) are compared with curves plotted for the AVI (green lines) and curves plotted for the Lindegaard Index (gray lines). The highest accuracy to detect MCA vasospasm was found for the AVI using both the mean and the peak systolic BFVs. BVR = basal vein of Rosenthal. Figure is available in color online only.

caused not only by vasospasm. Other factors influencing cerebral perfusion, such as the hematocrit level, the arterial $\mathrm{CO}_{2}$ content, intracranial pressure (ICP), and arterial blood pressure, might play an additional role. ${ }^{5}$ Especially in patients with impaired cerebral autoregulation, systemic blood pressure changes directly affect the MCA BFV. ${ }^{18}$ This illustrates the difficulty in defining clear cutoff values for BFV that indicate the presence vasospasm with high specificity and sensitivity. In one study, increasing the threshold for vasospasm in the MCA from $140 \mathrm{~cm} /$ sec to $220 \mathrm{~cm} / \mathrm{sec}$ (PSV) improved the specificity from $63 \%$ to $99 \%$, whereas the sensitivity decreased from $93 \%$ to $50 \% .^{10}$ The low PPV for vasospasm in patients with $\operatorname{BFV}\left(\mathrm{V}_{\text {mean }}\right)$ in the MCA between 120 and $199 \mathrm{~cm} / \mathrm{sec}$ confirms the high percentage of false-positive findings. ${ }^{23}$ Therefore, hyperperfusion alone or concomitant with vasospasm are relevant conditions in the differential diagnosis in patients with a mild to moderate increase in BFV, making additional Doppler criteria for vasospasm assessment desirable. Alternative parameters, such as the relative increase in BFV or differences in BFV between the left and right MCAs, might reveal a better correlation with symptomatic vasospasm, ${ }^{12}$ although contrary results have also been reported. ${ }^{23} \mathrm{~A}$ decline in the pulsatility index might predict symptomatic vasospasm, but it does not correlate with angiographic vasospasm..$^{15}$ In the original TCD study, the Lindegaard Index (the most common additional Doppler parameter) showed better agreement with angiographic spasm than the absolute BFV of the MCA. ${ }^{8}$ These findings, however, were challenged by subsequent studies. ${ }^{23}$ Few data exist for which the reliability of the Lindegaard Index using TCCS and extracranial duplex sonography have been analyzed. An equivalent accuracy for angiographic detection of vasospasm was found for PSV measurements in the MCA and calculation of the Lindegaard Index. ${ }^{6}$
Our study is important for 2 main reasons. First, regarding absolute $\mathrm{BFV}$, our data confirm that a threshold of $120 \mathrm{~cm} / \mathrm{sec}$ for the $\mathrm{V}_{\text {mean }}$ and $200 \mathrm{~cm} / \mathrm{sec}$ for the PSV provide the greatest accuracy for detecting vasospasm., ${ }^{1,3,10,15}$ Compared with a meta-analysis of 7 TCD ultrasonography studies using thresholds between 120 and $140 \mathrm{~cm} / \mathrm{sec}$ for the $V_{\text {mean }}$, the overall accuracy of our data are similar, showing a lower specificity but higher sensitivity. ${ }^{9}$ This means that our study contains a relatively large number of patients with false-positive findings but very few patients with false-negative findings, indicating again the relevance of hyperperfusion. Indeed, the AVI provided an increase in specificity and PPV for vasospasm detection without worsening of sensitivity or NPV. Our results suggest that there is slightly greater accuracy of the AVI than the absolute BFV in the MCA. As assumed, the AVI might discriminate between vasospasm and hyperperfusion better than BFV in the MCA alone. However, the AVI in our study failed to reduce the rate of false-positive findings so

TABLE 1. Comparison of the different TCCS parameters in comparison with DSA parameters

\begin{tabular}{lccccc}
\hline \multicolumn{1}{c}{ Parameter } & Accuracy & Sensitivity & Specificity & PPV & NPV \\
\hline MCA (PSV) & $83 \%$ & $78 \%$ & $86 \%$ & $77 \%$ & $87 \%$ \\
\hline MCA $\left(\mathrm{V}_{\text {mean }}\right)$ & $84 \%$ & $84 \%$ & $84 \%$ & $76 \%$ & $90 \%$ \\
\hline LI (PSV) & $80 \%$ & $73 \%$ & $84 \%$ & $74 \%$ & $83 \%$ \\
\hline LI ( $\left.\mathrm{V}_{\text {mean }}\right)$ & $84 \%$ & $86 \%$ & $82 \%$ & $75 \%$ & $90 \%$ \\
\hline AVI (PSV) & $86 \%$ & $83 \%$ & $88 \%$ & $81 \%$ & $89 \%$ \\
\hline AVI ( mean $\left._{\text {m }}\right)$ & $87 \%$ & $84 \%$ & $89 \%$ & $83 \%$ & $90 \%$ \\
\hline MCA+AVI (PSV) & $84 \%$ & $70 \%$ & $93 \%$ & $86 \%$ & $84 \%$ \\
\hline MCA+AVI ( mean $\left._{\text {m }}\right)$ & $88 \%$ & $78 \%$ & $94 \%$ & $88 \%$ & $88 \%$ \\
\hline
\end{tabular}

$\mathrm{LI}=\mathrm{Lindegaard}$ Index $; \mathrm{V}_{\text {mean }}=$ mean time-averaged maximum BFV. 
that optimal results for the PPV and the specificity could be obtained. The additional assessment of a high AVI value might help to confirm suspected vasospasm, whereas a low AVI value might suggest that signs of concomitant hyperperfusion should be further examined.

The second main finding is the superiority of the AVI in comparison with the Lindegaard Index. The high failure rate of the Lindegaard Index in our study was caused by the difficulty to reliably assess BFV in the extracranial ICA using a linear-array transducer. Strict limitations of head rotation in combination with a central line in the internal jugular vein and plasters impeded sufficient access to the ICA with an accurate angle correction. Furthermore, the assessment of the Lindegaard Index is more complex because of the additional insonation in the neck region by a second ultrasound probe. The alternative idea to assess the BFV of the ICA using the smaller transcranial 2-MHz probe at the mandibular angle does not enable angle correction and to date has not been evaluated. On the other hand, the low failure rate of AVI assessment confirmed the stable anatomy and high detection rate of the basal vein of Rosenthal by TCCS..$^{19}$ Nevertheless, the direct comparison between the AVI and the Lindegaard Index of all successful investigations revealed a higher accuracy of the AVI.

Few data exist regarding intracranial venous BFV after SAH. There are 2 small observational TCCS studies that have analyzed intracranial venous hemodynamics after SAH with regard to the patient's clinical outcome. One study described a positive correlation between the BFV in the transverse sinus and ICP. This was interpreted as secondary blood pooling toward the larger venous vessels due to stasis within the bridging veins caused by the increased ICP. ${ }^{13}$ A further increase in venous BFV occurs with the progressive compression of the venous system. The BFV in the basal vein of Rosenthal was not analyzed in this study, making a comparison with our results difficult. However, a significant increase in ICP can be ruled out in our patients, as all patients at high risk for critical ICP were monitored and treated using external ventricular drainage. Another study found a close relationship between BFV in the basal vein of Rosenthal and global CBF after SAH. The BFV in the MCA, however, correlated less significantly with the CBF." ${ }^{11}$ Furthermore, patients with poor outcomes exhibited a decrease in BFV in the basal vein of Rosenthal during vasospasm, whereas a favorable outcome was associated with an increase in venous BFV. As patients with good clinical outcome after SAH tend to have higher cerebral perfusion, an increased venous BFV was interpreted to represent elevated CBF. These data indirectly support our assumption that hyperperfusion leads to decreases in the AVI, whereas vasospasm leads to an opposite effect. In this study, DSA was not performed during the critical time frame of vasospasm. In our study, the degree of vasospasm did not influence the venous BFV, supporting the assumption that the high AVI in patients with vasospasm is exclusively caused by the increased arterial BFV.

The relatively small number of patients $(n=102)$ investigated in our study naturally does not allow a concluding judgment regarding the value of the AVI, especially as some limitations must be discussed. We hypothesized an unchangeable diameter and cross-sectional area of the basal vein of Rosenthal and therefore a direct correlation between BFV and perfusion. A reduction in the venous vessel lumen during arterial vasospasm in patients with normal ICP has not been described in the literature. However, a passive increase in the vessel diameter due to the increased CBF cannot be ruled out. This might reduce the increase in BFV in the basal vein of Rosenthal during hyperperfusion, with negative consequences for the PPV and specificity of the AVI.

Another objection regarding the AVI might be that the basal vein of Rosenthal does not completely drain the blood from the MCA territory. Changes in BFV due to hyperperfusion, however, have a global character and are not restricted to the MCA territory. ${ }^{11}$

A further limitation of our study is the time interval of up to 24 hours between angiography and ultrasonography. The possibility cannot be completely excluded that changes in vessel diameters or changes in global cerebral perfusion occurred between the studies. Vasospasm detected by DSA within the critical phase generally lasts for at least several days, making false-negative TCCS results unlikely.

Finally, the AVI evaluated in our study refers only to vasospasm in the MCA. However, vasospasm in the MCA normally has a greater clinical significance, and its assessment by TCCS is more reliable than assessment by TCCS in other basal cerebral arteries. The direct comparison with the Lindegaard Index was another reason to focus on vasospasm of the MCA.

\section{Conclusions}

We have proposed and evaluated a new diagnostic parameter for vasospasm analysis with ultrasonography. The AVI seems to be a reliable approach in addition to assessment of absolute BFV in the MCA to improve the diagnostic accuracy of TCCS. Furthermore, the AVI showed better practicability and a greater reliability than the established Lindegaard Index and might replace the latter in the assessment of vasospasm using TCCS.

\section{References}

1. Aaslid R, Huber P, Nornes H: Evaluation of cerebrovascular spasm with transcranial Doppler ultrasound. J Neurosurg 60:37-41, 1984

2. Egge A, Sjøholm H, Waterloo K, Solberg T, Ingebrigtsen T, Romner B: Serial single-photon emission computed tomographic and transcranial doppler measurements for evaluation of vasospasm after aneurysmal subarachnoid hemorrhage. Neurosurgery 57:237-242, 2005

3. Grolimund P, Seiler RW, Aaslid R, Huber P, Zurbruegg H: Evaluation of cerebrovascular disease by combined extracranial and transcranial Doppler sonography. Experience in 1,039 patients. Stroke 18:1018-1024, 1987

4. Kincaid MS, Souter MJ, Treggiari MM, Yanez ND, Moore A, Lam AM: Accuracy of transcranial Doppler ultrasonography and single-photon emission computed tomography in the diagnosis of angiographically demonstrated cerebral vasospasm. J Neurosurg 110:67-72, 2009

5. Klingelhöfer J, Dander D, Holzgraefe M, Bischoff C, Conrad B: Cerebral vasospasm evaluated by transcranial Doppler ultrasonography at different intracranial pressures. J Neurosurg 75:752-758, 1991

6. Krejza J, Kochanowicz J, Mariak Z, Lewko J, Melhem ER: 
Middle cerebral artery spasm after subarachnoid hemorrhage: detection with transcranial color-coded duplex US. Radiology 236:621-629, 2005

7. Krejza J, Mariak Z, Lewko J: Standardization of flow velocities with respect to age and sex improves the accuracy of transcranial color Doppler sonography of middle cerebral artery spasm. AJR Am J Roentgenol 181:245-252, 2003

8. Lindegaard KF, Nornes H, Bakke SJ, Sorteberg W, Nakstad P: Cerebral vasospasm diagnosis by means of angiography and blood velocity measurements. Acta Neurochir (Wien) 100:12-24, 1989

9. Lysakowski C, Walder B, Costanza MC, Tramèr MR: Transcranial Doppler versus angiography in patients with vasospasm due to a ruptured cerebral aneurysm: a systematic review. Stroke 32:2292-2298, 2001

10. Mariak Z, Krejza J, Swiercz M, Kordecki K, Lewko J: Accuracy of transcranial color Doppler ultrasonography in the diagnosis of middle cerebral artery spasm determined by receiver operating characteristic analysis. J Neurosurg 96:323-330, 2002

11. Mursch K, Wachter A, Radke K, Buhre W, Al-Sufi S, Munzel $\mathrm{U}$, et al: Blood flow velocities in the basal vein after subarachnoid haemorrhage. A prospective study using transcranial duplex sonography. Acta Neurochir (Wien) 143:793800,2001

12. Naval NS, Thomas CE, Urrutia VC: Relative changes in flow velocities in vasospasm after subarachnoid hemorrhage: a transcranial Doppler study. Neurocrit Care 2:133-140, 2005

13. Niesen WD, Rosenkranz M, Schummer W, Weiller C, Sliwka U: Cerebral venous flow velocity predicts poor outcome in subarachnoid hemorrhage. Stroke 35:1873-1878, 2004

14. Proust F, Callonec F, Clavier E, Lestrat JP, Hannequin D, Thiébot J, et al: Usefulness of transcranial color-coded sonography in the diagnosis of cerebral vasospasm. Stroke 30:1091-1098, 1999

15. Rajajee V, Fletcher JJ, Pandey AS, Gemmete JJ, Chaudhary $\mathrm{N}$, Jacobs TL, et al: Low pulsatility index on transcranial Doppler predicts symptomatic large-vessel vasospasm after aneurysmal subarachnoid hemorrhage. Neurosurgery 70:1195-1206, 2012

16. Saito I, Shigeno T, Aritake K, Tanishima T, Sano K: Vasospasm assessed by angiography and computerized tomography. J Neurosurg 51:466-475, 1979

17. Sloan MA, Haley EC Jr, Kassell NF, Henry ML, Stewart SR, Beskin RR, et al: Sensitivity and specificity of transcranial Doppler ultrasonography in the diagnosis of vasospasm following subarachnoid hemorrhage. Neurology 39:1514-1518, 1989

18. Soehle M, Czosnyka M, Pickard JD, Kirkpatrick PJ: Continu- ous assessment of cerebral autoregulation in subarachnoid hemorrhage. Anesth Analg 98:1133-1139, 2004

19. Stolz E, Kaps M, Kern A, Babacan SS, Dorndorf W: Transcranial color-coded duplex sonography of intracranial veins and sinuses in adults. Reference data from 130 volunteers. Stroke 30:1070-1075, 1999

20. Swiat M, Weigele J, Hurst RW, Kasner SE, Pawlak M, Arkuszewski M, et al: Middle cerebral artery vasospasm: transcranial color-coded duplex sonography versus conventional nonimaging transcranial Doppler sonography. Crit Care Med 37:963-968, 2009

21. Valdueza JM, Schmierer K, Mehraein S, Einhäupl KM: Assessment of normal flow velocity in basal cerebral veins. A transcranial doppler ultrasound study. Stroke 27:1221-1225, 1996

22. Valdueza JM, Schreiber SJ, Röhl JE, Klingebiel R: Neurosonology and Neuroimaging of Stroke. Stuttgart: Thieme, 2008, pp 30-44

23. Vora YY, Suarez-Almazor M, Steinke DE, Martin ML, Findlay JM: Role of transcranial Doppler monitoring in the diagnosis of cerebral vasospasm after subarachnoid hemorrhage. Neurosurgery 44:1237-1248, 1999

24. Wang HC, Lin WC, Yang TM, Chen WF, Lin YJ, Tsai NW, et al: Time course of cerebral hemodynamics in aneurysmal subarachnoid hemorrhage. J Clin Ultrasound 40:91-98, 2012

25. White H, Venkatesh B: Applications of transcranial Doppler in the ICU: a review. Intensive Care Med 32:981-994, 2006

\section{Disclosures}

The authors report no conflict of interest concerning the materials or methods used in this study or the findings specified in this paper.

\section{Author Contributions}

Conception and design: Connolly, Valdueza. Acquisition of data: Connolly. Analysis and interpretation of data: Connolly, Schreiber, Leithner, Bohner, Valdueza. Drafting the article: Connolly, Schreiber, Leithner, Bohner. Critically revising the article: Schreiber, Leithner, Bohner, Vajkoczy, Valdueza. Approved the final version of the manuscript on behalf of all authors: Connolly. Statistical analysis: Connolly, Schreiber.

\section{Correspondence}

Florian Connolly, Department of Neurology, University Hospital Charité, Augustenburger Platz 1, Berlin 13344, Germany. email: florian.connolly@charite.de. 